\title{
A Comparative Study on the Inter-Session and Inter- Examiner Reliability of Corneal Power Measurement Using Various Keratometry Instruments
}

\author{
Md Muziman Syah MM ${ }^{\mathrm{a}, \mathrm{b}}$, Mutalib $\mathrm{HA}^{\mathrm{a}}$, Sharanjeet Kaur MS ${ }^{\mathrm{a}}$, Khairidzan Khairidzan MK \\ ${ }^{a}$ Programme of Optometry and Visual Science, Faculty of Health Sciences, Universiti Kebangsaan Malaysia. \\ ${ }^{b}$ Department of Optometry and Visual Science, Kulliyyah of Allied Health Sciences, International Islamic University Malaysia. \\ 'Department of Ophthalmology, Kulliyyah of Medicine, International Islamic University Malaysia.
}

\begin{abstract}
Introduction: The purpose of this study was to evaluate inter-session repeatability, inter-examiner reproducibility and inter-device agreement of corneal power measurements from manual keratometer, autokeratometer, topographer, Pentacam high resolution and IOLMaster. Methods: Two sets of mean corneal power measurements $(n=40)$ were compared for inter-session repeatability and inter-examiner reproducibility in each instrument. Repeatability and reproducibility were evaluated by within-subject standard deviation (Sw), coefficient of variation (COV) and intraclass correlation coefficient (ICC). A oneway repeated measures analysis of variance was conducted to compare differences in the corneal power between each instrument pair. The Bland and Altman analysis and Pearson's correlation were employed to assess agreement and determine strength of relationship between measurements. Results: There were no significant differences in mean corneal power measurements between 2 different visits $(p>0.05)$. The Sw and COV values between 2 visits were lower than $0.09 \mathrm{D}$ and $0.20 \%$ respectively. The ICCs were stronger than 0.99 in all instruments. For reproducibility of each instrument, differences of the measurements between 2 different examiners were also insignificant $(p>0.05)$. The $\mathrm{Sw}$ and Cov values between 2 examiners were lower than $0.11 \mathrm{D}$ and $0.23 \%$ respectively. The ICCs were 0.99 and above in all instruments. The 95\% limit of agreement between instruments ranged from -0.29 to $1.13 \mathrm{D}$ and the $r$-values were stronger than 0.84. Conclusion: The corneal power measurements using these 5 instruments were repeatable and reproducible. These instruments can also be used interchangeably, however the topographer should be used with caution.
\end{abstract}

KEYWORDS: Inter-session repeatability, inter-examiner reproducibility, inter-device agreement, corneal power.

\section{INTRODUCTION}

Reliable and accurate corneal power measurement determines intraocular lens (IOL) power calculation for patients who are undergoing cataract surgery. The inaccurate corneal power measurement leads to a false IOL power which causes unintentional postoperative ametropia and reduced vision after cataract surgery. In standard clinical setting,

Corresponding author:

Md Muziman Syah Md Mustafa, PhD

Programme of Optometry and Visual Science,

School of Healthcare Sciences,

Faculty of Health Sciences,

Universiti Kebangsaan Malaysia,

Jalan Raja Muda Abdul Aziz,

50300 Kuala Lumpur, Malaysia.

Tel: 603-9289 7495

Fax: 603-2691 0488

Email: phd.optom@gmail.com corneal power is usually determined using conventional keratometer or topographer. These instruments measure the anterior corneal surface at 3.0 $\mathrm{mm}$ diameter of paracentral cornea and use a 1.3375 keratometric index in calculating the total corneal power from the anterior corneal measurements. ${ }^{1-2}$ Several studies have discussed about the repeatability and agreement of corneal power measurement between manual keratometer, autokeratometer and corneal topographer. ${ }^{2-6}$ However, most of the previous studies only compared repeatability of the corneal power within the measurements in the same visit (intra-session repeatability), and to the best of our knowledge, no studies had evaluated the inter-device agreement of corneal power measurements between the Pentacam high resolution to a manual keratometer. The aim of this study was to assess the inter-session and inter-examiner reliability, and inter-device agreement of corneal power obtained from manual keratometer, autokeratometer, corneal topographer, Pentacam high resolution and IOLMaster. 


\section{MATERIAL AND METHODS}

This study was a comparative cross sectional study. Prospectively, forty healthy subjects were recruited at Optometry Clinic and Eye Specialist Clinic, International Islamic University Malaysia. The inclusion criteria were 1) no corneal or ocular diseases, 2) no history of trauma or ocular surgery, 3) able to fixate at examination target, 4) best corrected visual acuity (BCVA) of better than 6/9, 5) refractive errors were less than $4.00 \mathrm{DS}$ and/or 2.00 DC, and 6) free from contact lens wearing for at least 8 hours.

The purpose, benefits, risks, and procedures involved were explained to all subjects. A written consent form in accordance with institutional guidelines and the tenets of the Declaration of Helsinki was signed by each subject. This research was approved by the Ethical Committee, Pusat Perubatan Universiti Kebangsaan Malaysia, UKM (Ref: UKM1.21.3/244/NN-056-2013).

\section{Measurements}

The corneal powers were evaluated using the Topcon OM-4 manual keratometer, Japan (manual keratometer), Grand Seiko WR-5100K binocular autokeratometer, Japan (autokeratometer), Shin Nippon CT-1000 topographer, Japan (topographer), Oculus Pentacam high resolution, Germany (Pentacam HR) and Carl Zeiss IOLMaster 500, Germany (IOLMaster). All of these keratometry instruments use a similar keratometric index of 1.3375 in converting anterior corneal power measurement into an estimated of total corneal power.

Manual keratometer and autokeratometer are commonly available keratometry instruments in optometry and ophthalmology clinical setting. The keratometers measure the paracentral corneal power at a diameter of approximately $3.0 \mathrm{~mm}$. For the Grand Seiko autokeratometer, both eyes were opened during the measurement process. The Shin Nippon topographer has Placido disk of 20 rings with 6292 measuring points. The measurement zone of this topographer is within $0.5 \mathrm{~mm}$ to $10.6 \mathrm{~mm}$ diameter. It provides simulated keratometric (sim-K) values along the steepest and flattest meridians at the $3.0 \mathrm{~mm}$ zone.

The Pentacam HR uses a rotating measurement principle by Scheimpflug camera and a scanning illumination slit. The camera and scanning slit light rotate 360 degrees around the eye in 1 second to produce completely 50 images with 2760 measuring points per recorded full Scheimpflug image. For the Pentacam HR, this study considered a sim-K measurement produced along the steepest and flattest meridians at the $3.0 \mathrm{~mm}$ central zone. The IOLMaster is a non-contact optical coherence tomography that uses optical imaging technique with infrared laser light.

The instrument measures corneal power by analyzing data from a hexagonal array of 6 points of light reflected off the surface of the cornea at a diameter of $2.5 \mathrm{~mm}$. The corneal power measurement was initially performed using the manual keratometer, followed by the autokeratometer, topographer, Pentacam HR and IOLMaster. The sequence was maintained throughout this study.

One eye of each subject with better BCVA was measured in this study. A total of 3 measurements for each instrument was performed in a visit to obtain the mean of the measurements. The measurement of the corneal power was performed by two examiners. In first visit, both examiner performed the measurement. After a week, one examiners repeated the same measurement on the same eyes. Both examiners had similar experience using these 5 instruments and they were masked throughout this study. The subjects were asked to blink completely just before each measurement.

\section{Statistical Analysis}

All the data obtained were analyzed using Statistical Package for the Social Sciences (SPSS) software version 20.0 and MedCalc software version 12.3 for Windows. Data normality were tested using ShapiroWilk test because of the sample size was less than 100 subjects. For each measurement, the corneal power for the horizontal meridian $\left(K_{\mathrm{h}}\right)$ and vertical meridian $\left(K_{\mathrm{v}}\right)$ were averaged $\left(\left[K_{\mathrm{h}}+K_{\mathrm{v}}\right] / 2=K_{\mathrm{ave}}\right)$. These values were calculated for 3 separate measurements. The figures were averaged manually or automatically by the instruments to obtain the mean average corneal power.

This study evaluated two faces of reliability which were inter-session repeatability and interexaminer reproducibility. ${ }^{7}$ The repeatability and reproducibility were assessed by the within-subject standard deviation (Sw), coefficient of variation (COV) and intraclass coefficient (ICC). The COV is the Sw divided by the mean between the two visits or two examiners. A lower COV indicates higher repeatability or reproducibility. The ICC with a twoway mixed model and consistency type, and twoway random model and absolute agreement type were employed to evaluate the repeatability and reproducibility respectively. An ICC value of $\geq 0.90$ indicates good repeatability and reproducibility for clinical setting. $^{8}$ 
A one-way repeated measures analysis of variance at significant level of 0.05 was conducted to determine differences in the corneal powers measured between instruments. The Pearson's correlation coefficient ( $r$-value) was also calculated to assess the strength of linear relationship between instruments. A $r$-value of $>0.90$ indicates excellent strenght of correlation. The inter-device agreement between instruments were assessed using BlandAltman analysis by comparing the range of $95 \%$ limit of agreement (LOA). The LOA is defined as the mean difference \pm 1.96 multiplied by the standard deviation of the difference to provide an interval within which $95 \%$ of the differences between the two instruments. A lower LOA indicates higher agreement.

\section{RESULTS}

Forty eyes of 40 subjects have undergone 2 sets of corneal power measurements. The mean age of the subjects was $22.02 \pm 1.11$ years ranging from 20 to 25 years. The subjects consisted of 14 males (35\%) and 26 females (65\%). All subjects recruited were Malays (39 subjects or $97.5 \%$ ) and one was Arabic (1 subject or 2.5\%). The distributions of mean average corneal power measurements were normally distributed based on Shapiro-Wilk normality test result, $p>0.05$.

Inter-Session Repeatability and Inter-Examiner Reproducibility

All Sw values and COVs of inter-session repeatability and inter-examiner reproducibility were less than $0.09 \mathrm{D}$ and $0.20 \%$ respectively in all instruments except for the reproducibility of the topographer. The ICCs were at least 0.99 in all instruments. Thus, the ICC values indicated that all instruments achieved the repeatability and reproducibility levels for clinical setting.

The IOLMaster had the highest level of repeatability and reproducibility among the compared instruments. It was followed by the autokeratometer, Pentacam HR, manual keratometer and topographer (Table I). However, there were no significant differences of mean average corneal power measurement between the 2 consecutive visits and between the 2 examiners for each instrument $(p>0.05)$.

Table I. Results for inter-session repeatability and inter-examiner reproducibility of corneal power measurements

\begin{tabular}{lllllll}
\hline \multirow{2}{*}{ Instruments } & \multicolumn{2}{l}{ Inter-Session Repeatability } & \multicolumn{2}{l}{ Inter-Examiner Reproducibility } \\
\cline { 2 - 7 } & SW (D) & COV (\%) & ICC & SW (D) & COV (\%) & ICC \\
\hline Manual Keratometer & 0.06 & 0.13 & 0.996 & 0.06 & 0.13 & 0.996 \\
Autokeratometer & 0.03 & 0.06 & 0.999 & 0.03 & 0.07 & 0.999 \\
Topographer & 0.08 & 0.19 & 0.991 & 0.10 & 0.22 & 0.989 \\
Pentacam HR & 0.04 & 0.09 & 0.998 & 0.04 & 0.09 & 0.998 \\
IOLMaster & 0.02 & 0.06 & 0.999 & 0.02 & 0.05 & 0.999 \\
\hline
\end{tabular}

\section{Inter-Device Agreement}

The mean corneal power measurement for manual keratometer, autokeratometer, topographer, Pentacam HR and IOLMaster were $43.75 \pm 0.91 \mathrm{D}$, $43.83 \pm 0.94 \mathrm{D}, 43.67 \pm 0.86 \mathrm{D}, 43.79 \pm 0.95 \mathrm{D}$ and $43.86 \pm 0.91 \mathrm{D}$ respectively. There were no statistically significant differences in measuring corneal power between devices $(p>0.05)$. However, the $r$-values of correlation and the 95\% LOAs between the instruments which involved the topographer were lower than 0.90 and out of \pm 1.00 D respectively. The other paired comparisons showed that the instruments excellently correlated and agreeable to each other in measuring corneal power. The result of Bland-Altman analysis revealed that the range of $95 \%$ LOA between the autokeratometer and IOLMaster was smaller than the range of $95 \%$ LOA found in the other instrument pairs (Table II). 
Table II. Results of Pearson's correlation and Bland-Altman analysis of mean corneal power measurement between instruments

\begin{tabular}{llll}
\hline Compared Instruments & ${ }^{a}$ r-Value & Lower 95\% LOA (D) & Upper 95\% LOA (D) \\
\hline Manual Keratometer vs Autokeratometer & 0.951 & -0.65 & 0.50 \\
Manual Keratometer vs Topographer & 0.856 & -0.85 & 1.01 \\
Manual Keratometer vs Pentacam HR & 0.981 & -0.40 & 0.33 \\
Manual Keratometer vs IOLMaster & 0.960 & -0.61 & 0.39 \\
Autokeratometer vs Topographer & 0.853 & -0.82 & 1.13 \\
Autokeratometer vs Pentacam HR & 0.952 & -0.53 & 0.62 \\
Autokeratometer vs IOLMaster & 0.991 & -0.29 & 0.22 \\
Topographer vs Pentacam HR & 0.881 & -1.00 & 0.77 \\
Topographer vs IOLMaster & 0.844 & -1.16 & 0.78 \\
Pentacam HR vs IOLMaster & 0.958 & -0.61 & 0.46 \\
\hline
\end{tabular}

${ }^{a}$ All instruments were significantly correlated with each other $(p<0.001)$.

Bold - The instruments agreement between each other were out of clinical acceptance range; $\geq$ $\pm 1.00 \mathrm{D}$.

\section{DISCUSSION}

A precise corneal power measurement is essential in optometric and ophthalmology management. For examples, change of cornea power measurement gives information in detection and monitoring the progression of corneal diseases ${ }^{9}$ and inaccuracy of corneal power measurement after laser refractive surgery is the main factor in $\mathrm{IOL}$ power miscalculation. ${ }^{10-11}$

All of the 5 instruments showed a highly repeatability and reproducibility in measuring corneal power. Similar to this present study, Shirayama et al. $^{2}$ and Karabatsas et al. ${ }^{12}$ also revealed good repeatability and reproducibility with manual keratometer respectively. Our study demonstrated a higher repeatability of the Grand Seiko WR-5100K binocular autokeratometer compared to Davies and associates' report which used the same model of autokeratometer. ${ }^{3}$ This finding attributed to the measurements in the previous study performed on both eyes of the same subjects as they were dependent. We found that the Grand Seiko had excellent inter-examiner reproducibility which may suggest that the binocular viewing during measurement offers better subjects' cooperation. Huang et al. also demonstrated good repeatability and reproducibility of topographer. ${ }^{6}$ However, they reported slightly higher repeatability and reproducibility than those we obtained. The difference was observed as they used a new Hummel OphthaTOP which has higher resolution of 2.0-megapixel camera which projects 30 rings onto the cornea with 10800 measuring points $^{6}$, while in this study, Shin Nippon topographer projects 20 rings with 6292 measuring points only at one time. It indicates that having more measuring points of topography produce a more precise keratometry reading. On the other hand, the duration of topographer measurement is relatively longer than other 4 compared instruments in order to ensure measuring target sharp and in-focus for measurement. These explained why the topographer in our study provided the least precise measurement among the 5 compared instruments. In an intra-session repeatability study, McAlinden and colleagues demonstrated good repeatability of the Pentacam HR with used the same 50-image cornea fine scan mode in this current study. ${ }^{13}$ For the interexaminer reproducibility results, this study was found consistent to previous report by HernándezCamarena et al. ${ }^{14}$ This present study revealed that the IOLMaster tends to provide lower SW and COV, and also higher ICC for both repeatability and reproducibility.

These findings were in line with earlier reports by Shirayama et al. ${ }^{3}$ and Wang et al. ${ }^{5}$ We believed that the IOLMaster provides more precise measurement because its modality only acquires an image by 6 light spots in hexagonal pattern and it is relatively easy to be administered.

Based on the results of the corneal power measurements obtained from the 5 different instruments, the topographer pairs of differences between the other 4 instruments were found to be clinically significant, where the differences magnitude were $1.00 \mathrm{D}$ or more. These findings were supported by a few previous studies, where their results demonstrated a statistically significant different for topographer in comparison to manual keratometer, IOLMaster, ${ }^{2,5}$ autokeratometer ${ }^{5}$ and Pentacam HR..$^{15}$ On the contrary, a previous report by Wang et al., the topography measurement using the Allegro topographer (used 20 Placido-disc rings with 22000 measuring point) was similar to autokeratometer and the Pentacam HR. ${ }^{5}$ 
Thus, we postulated that the Shin Nippon topographer has low ability to capture reproducible data that leads to least agreement to the other keratometry instruments.

Previous investigation by Davies et al. reported that the Javal-Schiotz, a manual keratometer that uses fixed doubling system had good agreement with the Grand Seiko autokeratometer. ${ }^{3}$ Although this present study used a 1-position keratometer that incorporates variable doubling system, the findings were in line with those reported in Davies et al. Therefore, it could suggest that the doubling system in a manual keratometer does not affect the interdevice agreement with other keratometry instruments. Wang and associates also reported that the Pentacam HR was agreeable with autokeratometer. ${ }^{5}$ No previous works had been carried out on inter-device agreement between the Pentacam HR and a manual keratometer. In a previous study used basic Pentacam model, the Pentacam was not agreeable to manual keratometer. ${ }^{16}$ On the contrast, this present study found that the Pentacam HR had good inter-device agreement to the manual keratometer. We assumed that these differences attributed to the Pentacam HR which measures at 2760 measuring points per image, whereas the basic Pentacam model only measures at 500 measuring points per image.

Several studies also reported that the IOLMaster produced slightly steeper corneal power measurement than the manual keratometer ${ }^{2}$, autokeratometer ${ }^{5}$ and Pentacam $\mathrm{HR}^{5}$, but the differences were not clinically significant, and they were agreeable to each other. It could be postulated that the higher measurement in the IOLMaster is because of its analyses is smaller at central corneal area with a diameter of $2.5 \mathrm{~mm}$ compared to the Pentacam HR, autokeratometer and manual keratometer which are approximately at $3.0 \mathrm{~mm}$ diameter. In a normal cornea, the curvature is steeper at central zone and it progressively flatter towards peripheral zone.

\section{Limitations}

The limitation of this study is that we have conducted the corneal power measurement only on healthy corneas. We did not compare other models from the same brand.

\section{CONCLUSIONS}

In conclusion, all of these instruments are highly repeatable and reproducible in measuring corneal power on normal corneas. All instruments can be used interchangeably in measuring corneal power, but the topographer should be used with caution as the correlation strength and 95\% LOA between other instruments were out of clinical acceptance.

\section{Declaration}

The authors have no financial interest in all instruments that have been used in this study.

\section{Acknowledgement}

This research is financially supported by the IIUM research funding (IIUM/202/C/1/1/4891).

\section{REFERENCES}

1. Rabbetts RB. Bannett and Rabbetts's clinical visual optics. $4^{\text {th }}$ ed. Edinburgh: Butterworth Heinemann Elseveir, 2007.

2. Shirayama M, Wang L, Weikert MP, Koch DD. Comparison of corneal powers obtained from 4 different devices. Am J Ophthalmol 2009; 148:528-35.

3. Davies LN, Mallen EAH, Wolffsohn JS, Gilmartin B. Clinical evaluation of the Shin Nippon NVision -K 5001/Grand Seiko WR-5100K autorefractor. Optom Vis Sci 2003; 80:320-4.

4. Kobashi H, Kamiya $\mathrm{K}$, Igarashi $\mathrm{A}$, et al. Comparison of corneal power, corneal astigmatism, and axis location in normal eyes obtained from an autokeratometer and a corneal topographer. J Cataract Refract Surg 2012; 38:648-54.

5. Wang Q, Savini G, Hoffer KJ, et al. A comprehensive assessment of the precision and agreement of anterior corneal power measurements obtained using 8 different devices. PLOS ONE. 2012; 7: e45607. doi:10.1371/journal.pone.0045607.

6. Huang J, Savini G, Chen H, et al. Precision and agreement of corneal power measurements obtained using a new corneal topographer OphthaTOP. PloS ONE. 2015; 10: e109414. doi:10.1371/journal.pone. 0109414 .

7. McAlinden C, Khadka J, Pesudovs $\mathrm{K}$, et al. Statistical methods for conducting agreement (comparison of clinical tests) and precision (repeatability or reproducibility) studies in optometry and ophthalmology. Ophthalmic Physiol Opt. 2011; 31: 330-8.

8. Portney LG \& Watkins MP. Foundation of clinical research applications to practice. $2^{\text {nd }}$ ed. New Jersey: Appleton \& Lange, 2000.

9. Kanski JJ, Bowling B. Clinical ophthalmology: a systematic approach. $7^{\text {th }}$ ed. Poland: Butterworth Heinemann Elseveir Ltd, 2011.

10. Jin H, Auffarth GU, Guo H, Zhao P. Corneal power estimation for intraocular lens power calculation after corneal laser refractive 
surgery in Chinese eyes. J Cataract Refract Surg 2012; 38:1749-57.

11. Yang R, Yeh A, George MR, et al. Comparison of intraocular lens power calculation methods after myopic laser refractive surgery with previous refractive surgery data. J Cataract Refract Surg 2013; 39:1327-35.

12. Karabatsas CH, Cook SD, Papaefthymiou J, Turner P, Sparrow JM. Clinical evaluation of keratometry and computerised videokeratography: intraobserver and interobserver variability on normal and astigmatic corneas. $\mathrm{Br} \mathrm{J}$ Ophthalmol 1998; 82:637-42.

13. McAlinden C, Khadka J, Pesudovs K, et al. A comprehensive evaluation of the precision (repeatability and reproducibility) of the Oculus Pentacam HR. Invest. Ophthalmol. Vis. Sci. 2011; 52(10): 7731-7737.

14. Hernández-Camarena JC, Chirinos-Saldaña $P$, Alejandro Navas A, et al. Repeatability, reproducibility, and agreement between three different Scheimpflug systems in measuring corneal and anterior segment biometry. J Refract Surg 2014; 30:616-21.

15. Hidalgo IR, Rozema JJ, Dhubhghaill SI, et al. Repeatability and inter-device agreement for three different methods of keratometry: Placido, Scheimpflug, and color LED corneal topography. J Refract Surg 2015; 31:176-81.

16. Uçakhan ÖÖ, Akbel V, Bıyıklı1 Z, Kanpolat A. Comparison of corneal curvature and anterior chamber depth measurements using the manual keratometer, Lenstar LS 900 and the Pentacam. Middle East Afr J Ophthalmol 2013; 20: 201-6. 